\title{
Immunoglobulin GM genes, cytomegalovirus immunoevasion, and the risk of glioma, neuroblastoma, and breast cancer
}

\author{
Janardan P. Pandey* \\ Department of Microbiology and Immunology, Medical University of South Carolina, Charleston, SC, USA
}

\section{Edited by:}

Charles Stringfellow Cobbs, Swedish

Neuroscience Institute, USA

\section{Reviewed by:}

James C. Neil, University of Glasgow, UK

Luisa Lanfrancone, European Institute of Oncology, Italy

*Correspondence:

Janardan P. Pandey, Department of Microbiology and Immunology,

Medical University of South Carolina, 173 Ashley Avenue, Charleston, SC 29425-2230, USA

e-mail:pandeyj@musc.edu
Human cytomegalovirus (HCMV), a common herpes virus, has been reported to be a risk factor for many diseases, including malignant diseases such as glioma, neuroblastoma, and breast cancer. Some of the HCMV-associated diseases (e.g., glioma) are rare. The question arises: how could a common virus be associated with uncommon diseases? Interactions between a major gene complex of the human immune system and a viral immunoevasion strategy - a probable mechanism of their co-evolutionary adaptation - may shed light on this paradox. To ensure its survival, HCMV has evolved sophisticated immunoevasion strategies. One strategy involves encoding decoy $F_{c} \gamma$ receptors ( $\left.F_{c} \gamma R\right)$, which may enable the virus to evade host immunosurveillance by avoiding the Fc $\gamma$-mediated effector consequences of anti-HCMV IgG antibody binding. Immunoglobulin G1 proteins expressing GM ( $\gamma$ marker) alleles 3 and 17 have differential affinity to the HCMV TRL11/IRL 11-encoded Fc $\gamma R$, and thus act as effect modifiers of HCMV-associated malignancies. The high affinity GM 3 allele has been shown to be a risk factor for neuroblastoma, glioma, and breast cancer. Additional studies involving other viral FcyRs as well as GM alleles expressed on other IgG subclasses are warranted.

\section{Keywords: GM allotypes, immunoevasion, cytomegalovirus, glioma, neuroblastoma, breast cancer}

\section{INTRODUCTION}

Immunoglobulin (Ig) GM ( $\gamma$ marker) allotypes are encoded by three very closely linked genes - Ig heavy chain G1 (IGHG1), IGHG2, and IGHG3 - on chromosome $14 \mathrm{q} 32$. There are currently 18 serologically testable GM specificities - four on $\gamma 1$ (1/a, $2 / \mathrm{x}$, $3 / \mathrm{f}, 17 / \mathrm{z})$, one on $\gamma 2(23 / \mathrm{n})$, and 13 on $\gamma 3(5 / \mathrm{b} 1,6 / \mathrm{c} 3,10 / \mathrm{b} 5$, 11/b0, 13/b3, 14/b4, 15/s, 16/t, 21/g1, 24/c5, 26/u, 27/v, 28/g5). Ig $\gamma 4$ chains do not express $\gamma 4$-specific unique allotypes, but they do express isoallotypes - determinants that behave as alleles in one IgG subclass (allotypes) but are also expressed in all molecules of at least one other subclass (isotypes). With the exception of allelic GM 3 and GM 17 determinants expressed in the Fd region, all other GM alleles are expressed in the Fc region of $\gamma$ chains (Figure 1). Linkage disequilibrium in the GM gene complex within a racial group is almost absolute and the determinants are transmitted as a group - haplotypes. Each major race has a distinct array of several GM haplotypes (1-3). There are also qualitative differences in the distribution of GM allotypes among various racial/ethnic groups. For instance, GM 3 is not commonly found in people of African descent or GM 6 in those of European ancestry; GM 1 is polymorphic only in people of European ancestry.

Population genetic properties of the GM gene complex marked differences in allele frequencies of among races, strong linkage disequilibrium within a race, and racially restricted occurrence of GM haplotypes - collectively suggest that differential selection over many generations may have played an important role in the maintenance of polymorphism at GM loci. As first suggested by Haldane (4) and recently emphasized by others (5), major infectious diseases have been the principal selective forces of natural selection in humans. GM allotypes, as likely targets of these selective forces, could contribute to the outcome of infection via allotype-restricted differential immunity to the infectious pathogens.

This mini review will discuss how GM alleles influence certain immunoevasion strategies of human cytomegalovirus (HCMV) and thus act as potential effect modifiers of some HCMVassociated malignant diseases. Other putative mechanisms of GM gene involvement in immunity to self and non-self antigens are also discussed.

\section{GM ALLELES AND CYTOMEGALOVIRUS IMMUNOEVASION}

Human cytomegalovirus has evolved highly sophisticated strategies for evading host immunosurveillance. One strategy involves generating proteins that have functional properties of the $\mathrm{Fc} \gamma$ receptor $(\mathrm{Fc} \gamma \mathrm{R})$ (6), which may enable the virus to evade host immunosurveillance by avoiding the effector consequences of anti-HCMV IgG antibody binding, such as antibody-dependent cellular cytotoxicity (ADCC), antibody-dependent cellular phagocytosis, and antibody-dependent complement-dependent cytotoxicity. The HCMV-encoded Fc $\gamma \mathrm{R}$ may interfere with $\mathrm{F} c \gamma$ mediated effector functions by bipolar bridging, in which the Fab part of the anti-HCMV IgG antibody (paratope) binds to its antigenic target (epitope) on the virus, whereas the $\mathrm{Fc} \gamma$ part of the antibody binds to the Fc $\gamma$ R-like binding site on the viral protein, thus offering survival advantage to the virus by sterically hindering the access of (cellular) Fc $\gamma \mathrm{R}$-expressing effector cells to the HCMV-infected cells. 


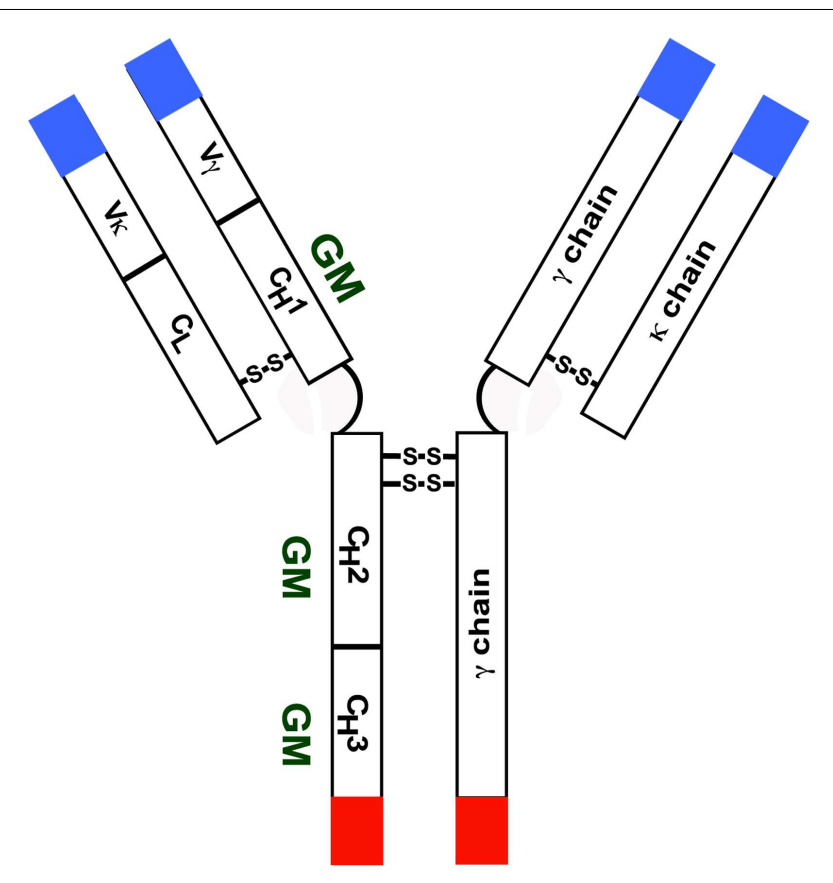

FIGURE 1 | Diagrammatic representation of an IgG molecule with a $\mathrm{k}$ light chain. Most $\mathrm{GM}$ alleles are expressed in the $\mathrm{CH} 2$ and $\mathrm{CH} 3$ domains.

Certain GM alleles appear to modulate this immunoevasion strategy of HCMV (7). We have shown that HCMV TRL11/IRL11encoded $F c \gamma R$ has significantly higher affinity for IgG1 proteins expressing the GM 3+,1-,2- allotypes than for those expressing the allelic GM $17+, 1+, 2+$ allotypes $(p=0.0005)$. Higher affinity of GM 3+,1-,2- expressing IgG1 to the TRL11-encoded Fc $\gamma R$ would imply that subjects with GM 3+,1-,2- allotypes would be more likely to have their Fc domains scavenged, thereby reducing their immunological competence to eliminate the virus through ADCC and other Fc-mediated effector mechanisms. Consequently, subjects possessing the GM $3+, 1-, 2-$ allotypes would be expected to be at an increased risk - while those carrying the GM $17+, 1+, 2+$ allotypes (because of the lower affinity to the viral $\mathrm{Fc} \gamma \mathrm{R}$ ) at a reduced risk - of developing HCMV-associated diseases. These results are reminiscent of those reported for the herpes simplex virus type 1 (HSV1)-encoded Fc $\gamma$ R; the binding pattern, however, is reversed: the HSV1-encoded Fc $\gamma \mathrm{R}$ binds much more strongly to the IgG molecule carrying the GM 1,17 allele than the one carrying the GM 3 allele (8). The contrasting binding patterns of the two viral Fc $\gamma$ Rs shed light on the nature of the evolutionary mechanism that maintains genetic polymorphism at the $\gamma 1$ locus. Since IgG antibodies expressing both alleles/haplotypes would be expected to be protective factors due to their modulating effects against the immunoevasion strategies of HCMV and HSV1, the heterozygotes at this locus would have advantage over homozygotes, resulting in the persistence of both alleles/haplotypes in the population. This would represent an example of balancing selection for the human adaptation of herpes viruses.
A brief review of the studies that suggest a role for GM genes as effect modifiers of HCMV-associated malignancies is presented below.

\section{CYTOMEGALOVIRUS, GLIOMAS, AND GM ALLELES}

Human cytomegalovirus is not considered an oncogenic virus at present, but several features of HCMV biology overlap with the essential alterations of cell physiology, including tumor microenvironment, that are hallmarks of cancer, as enunciated by Hanahan and Weinberg (9). In 2002, Cobbs and colleagues, for the first time, presented evidence that implicated this virus in gliomagenesis (10). Since then numerous studies have documented the presence of HCMV DNA, RNA, and protein in the majority of human gliomas, and HCMV-based therapies appear promising (11-13).

Although the current consensus in the field is that HCMV is an active promoter or oncomodulator of gliomagenesis, it is clear that among exposed individuals, not everyone is equally likely to develop HCMV-associated glioma, implying the presence of host genetic factors that might modulate immunity to this virus. Ig GM genes are good candidates, as they are involved in immunity to several viruses, including HCMV (14-23). These observations and especially the modulating influence of GM alleles on HCMV's immunoevasion strategies discussed above - led us to hypothesize that these polymorphic determinants might be risk factors for the development of glioma (24).

Using a case-control design, we tested this hypothesis in a group of 120 glioma patients and 133 random blood donors as controls. Both patients and controls were unrelated Caucasians from Portugal. DNA from the study subjects was typed for GM 3 and 17 alleles. Genotype frequencies were in Hardy-Weinberg equilibrium in both groups. Odds ratios (OR) for the GM genotypes for the risk of glioma were estimated by unconditional multivariate logistic regression analysis, adjusted for potential confounding variables (patient age and sex). Compared to subjects who were homozygous for the GM 17 allele, the GM 3 homozygotes were over twice as likely $(\mathrm{OR}=2.82)$, and the GM 3/17 heterozygotes were over three times as likely $(\mathrm{OR}=3.13)$ to develop glioma. Likewise, when comparing the combined GM 3/3 + 3/17 (i.e., GM 3 -carriers) genotypes to the GM 17/17 genotypes, the presence of the GM 3 allele conferred a significantly increased risk for glioma $(\mathrm{OR}=2.95)$ and high-grade astrocytoma $(\mathrm{OR}=3.11)$.

A multivariate Cox proportional hazard model, adjusted for patient age and sex, was used to investigate the association between GM genotypes and overall survival. Glioblastoma patients carrying the GM 17/17 genotype had longer median overall survival (35 months) than patients carrying the GM 3/17 or GM 3/3 genotypes (16 months), but the difference was not statistically significant, probably a reflection of small sample size $(\mathrm{HR}=2.78$, $p=0.19)$.

These results support the hypothesis that GM 3 and GM 17 alleles are risk factors for glioma (25). They also shed light on the possible reasons behind the racial differences in the prevalence of glioma. Age-adjusted glioma rates are considerably higher in Caucasians than in people of African descent. As noted earlier, the GM 3 allele is absent or extremely rare in people with unmixed African ancestry. 


\section{CYTOMEGALOVIRUS AND UNCOMMON GM GENOTYPES ASSOCIATED WITH NEUROBLASTOMA}

Almost four decades ago, two very unusual GM genotypes, $1+3+21-5+$ and $1+3+21+5-$, were shown to be highly significantly $(p<0.00005)$ associated with susceptibility to neuroblastoma, the most common extracranial solid tumor of childhood (26). The mechanism underlying this association has not yet been elucidated. However, a recent report documenting the expression of early and late HCMV proteins in primary neuroblastomas, neuroblastoma cell lines, and neuroblastoma xenografts (27) shed some light on the paradox: how a common virus (prevalence $\sim 80 \%$ ) could be associated with an uncommon (prevalence $\sim 0.025 \%$ ) disease. It is noteworthy that both uncommon genotypes associated with neuroblastoma include GM 3, the allele associated with high affinity to the HCMV TRL11/IRL11-encoded Fc $\gamma$ R. To gain further mechanistic insights into the GM gene involvement in the pathogenesis of this malignancy, it would be necessary to evaluate the affinity of viral $F c \gamma$ Rs to the IgG proteins expressing other GM alleles $-1,5$, and 21 - that constitute the genotypes associated with neuroblastoma.

\section{GM ALLELES, CYTOMEGALOVIRUS, AND THE RISK OF BREAST CANCER}

Using a matched case-control design, we have evaluated the contribution of GM alleles to the risk of breast cancer in a large ( 1710 subjects) multiethnic study population from Japan and Brazil (28). The study subjects were typed for several GM alleles $-\operatorname{IgG1}(3,17)$, $\operatorname{IgG} 2(23+/-)$, and $\operatorname{IgG} 3(5,21)$. Conditional logistic regression models were constructed to detect the association between GM alleles and breast cancer.

After adjusting for the potential confounders, the GM 3 allele of IgG1 was significantly associated with susceptibility to breast cancer in Caucasian subjects from Brazil $(p=0.0147)$; subjects possessing this allele were over twice as likely to develop breast cancer as those who lacked this allele $(\mathrm{OR}=2.07)$. No significant associations were found in other population groups. The reasons for racial differences in disease associations are not clear. Linkage disequilibrium between GM alleles in the Japanese is different from that in people of African or European descent, resulting in distinct arrays of GM haplotypes in various groups. It follows that linkage disequilibrium between any putative risk-conferring genes for breast cancer and GM alleles might also be different in these groups, contributing to the ethnic differences in genetic associations. Multiple genetic and non-genetic factors probably contribute to the risk of breast cancer, and racial differences in these factors may contribute to the differences in the observed associations. It is relevant to note that, of the four population groups examined, the frequency of the high affinity (to HCMVFc $\gamma$ R) GM 3 allele was the highest in the subjects of Caucasian descent (Brazil).

As in glioma and neuroblastoma, increasing evidence implicates HCMV in the etiopathogenesis of breast cancer. Evidence of viral expression has been found in over $97 \%$ of neoplastic breast epithelium (29). These findings were confirmed and extended in a recent investigation, which found $100 \%$ of the primary breast cancer samples HCMV positive, and also detected viral protein expression in neoplastic cells in sentinel lymph node metastases of breast cancer (30). Our finding of a significant association between the GM 3 allele and susceptibility to breast cancer, which is consistent with the predictions from the IgG1-HCMV-Fc $\gamma$ R binding studies (7), appears to unite the putative genetic and viral etiology of this malignancy.

\section{OTHER MECHANISMS OF GM GENE INVOLVEMENT IN CANCER}

So far, we have discussed how GM alleles could contribute to the risk of glioma, neuroblastoma, and breast cancer by modulating the viral immunoevasion strategies. However, this is unlikely to be the only mechanism underlying their involvement in these malignancies. These determinants could also contribute to the disease risk by influencing antibody responsiveness to the relevant viral and tumor-associated antigens. In a recent study, we found the levels of IgG antibodies to HCMV glycoprotein B to be the highest in GM 17/17 homozygotes, intermediate in GM 3/17 heterozygotes, and the lowest in GM 3/3 homozygotes (31). If anti-HCMV antibodies were protective, subjects with the low responder genotype - GM 3/3 - would be expected to be at a higher risk of developing HCMV-associated diseases than the subjects with the high responder GM 17/17 genotype, a prediction supported by our finding of GM 3 association with glioma and breast cancer discussed above. We have also reported contribution of GM alleles to humoral immunity to some tumor-associated antigens relevant to these malignancies, e.g., human epidermal growth factor receptor 2 (HER2) and mucin $1(32,33)$.

Although GM allotypes are expressed in the constant region of $\gamma$ chains, they could influence antibody specificity and affinity by imposing structural constraints (conformation) on the variable region. It is relevant to note that amino acid sequence polymorphism in the $\mathrm{CH} 1$ domain of the $\gamma 1$ chain - where the allelic determinants GM 3 and GM 17 are located - has been shown to modulate the kinetic competence of antigen binding sites (34). Another mechanism of GM gene involvement in cancer pathogenesis could involve ADCC, a major host immunosurveillance mechanism against tumors and virally infected cells. IgG antibody mediated $\mathrm{ADCC}$ is triggered upon ligation of $\mathrm{Fc} \gamma \mathrm{R}$ to the Fc region of IgG molecules, where the majority of GM alleles are expressed. It follows that genetic variation in $\mathrm{Fc} \gamma \mathrm{R}$ and $\mathrm{Fc}$ could contribute to the differences in the magnitude of ADCC. We have presented evidence of interactive effects of GM and Fc $\gamma$ RIIIa alleles on ADCC of cancer cells overexpressing HER1, HER2, mucin 1, and the disialoganglioside antigen GD2 (35-37).

\section{GM GENES AND GENOME-WIDE ASSOCIATION STUDIES}

Genome-wide association studies (GWAS) do not evaluate GM genes and, therefore, none of the GM gene-disease associations that were identified by hypothesis driven candidate gene approaches have been confirmed or refuted by these studies. GM genes are not included in the genotyping platforms commonly used in GWAS. Since these genes were not typed in the HapMap and 1000 Genomes projects, they cannot be imputed. Genes encoding IgG subclasses that harbor GM alleles are highly homologous and apparently not amenable to the high throughput genotyping technologies. This attribute may have contributed to their exclusion from the genotyping panels. 


\section{FUTURE STUDIES}

For a thorough understanding of the underlying immunogenetic mechanisms in HCMV-associated diseases, further studies are warranted. A brief outline of some of these follows. The IgGHCMV-Fc $\gamma \mathrm{R}$ binding studies discussed above employed the HCMV strain AD169 for cloning the TRL11 sequences. Whether or not TRL11-encoded Fc $\gamma \mathrm{R}$ from another strain would have the same affinity for allotypically disparate IgG1 proteins needs to be investigated. Additional studies to measure binding between allotypically different IgG2 and IgG3 proteins and HCMV TRL11- and UL119-encoded Fc $\gamma$ Rs are warranted. In this context, it would be interesting to evaluate the recently described HCMV RL13encoded Fc $\gamma \mathrm{R}$ (38) for its binding affinity to allotypically disparate IgG proteins.

In view of the strong linkage disequilibrium between particular GM alleles within a racial group, all GM alleles that constitute racially associated haplotypes must be evaluated to obtain a more complete understanding of the contribution of GM alleles to the risk of malignant diseases discussed above. Future studies should also consider examining possible interactive contribution of particular candidate genes to the risk of developing glioma, neuroblastoma, and breast cancer. Genes do not act in isolation: there is increasing evidence that epistasis - modification of the action of a gene by one or more other genes - plays a significant role in human diseases (39-41). GM and HLA are excellent candidate genes for investigations of epistasis in these malignancies, as both are targets of HCMV immunoevasion strategies $(7,42)$ and have been associated with the risk of these cancers $(25,26,28,43-$ 45). Results of these investigations may identify novel pathways to cancer immunity, which would be helpful in devising effective immunotherapeutic strategies against these malignancies.

\section{ACKNOWLEDGMENTS}

The author's laboratory is supported by grants and contracts from the Avon Foundation, the National Institutes of Health, and the Department of Defense.

\section{REFERENCES}

1. Grubb R. Advances in human immunoglobulin allotypes. Exp Clin Immunogenet (1995) 12:191-7.

2. Pandey JP, Li Z. The forgotten tale of immunoglobulin allotypes in cancer risk and treatment. Exp Hematol Oncol (2013) 2:6. doi:10.1186/2162-3619-2-6

3. Oxelius VA, Pandey JP. Human immunoglobulin constant heavy G chain (IGHG) $(\mathrm{Fc} \gamma)(\mathrm{GM})$ genes, defining innate variants of IgG molecules and B cells, have impact on disease and therapy. Clin Immunol (2013) 149:475-86. doi:10.1016/j.clim.2013.10.003

4. Lederberg J. J. B. S. Haldane (1949) on infectious disease and evolution. Genetics (1999) 153:1-3.

5. Karlsson EK, Kwiatkowski DP, Sabeti PC. Natural selection and infectious disease in human populations. Nat Rev Genet (2014) 15:379-93. doi:10.1038/nrg3734

6. Atalay R, Zimmermann A, Wagner M, Borst E, Benz C, Messerle M, et al. Identification and expression of human cytomegalovirus transcription units coding for two distinct Fcgamma receptor homologs. J Virol (2002) 76:8596-608. doi:10.1128/JVI.76.17.8596-8608.2002

7. Namboodiri AM, Pandey JP. The human cytomegalovirus TRL11/IRL11encoded $\mathrm{Fc} \gamma \mathrm{R}$ binds differentially to allelic variants of immunoglobulin G1. Arch Virol (2011) 156:907-10. doi:10.1007/s00705-011-0937-8

8. Atherton AK, Armour L, Bell S, Minson AC, Clark MR. The herpes simplex virus type $1 \mathrm{Fc}$ receptor discriminates between IgG1 allotypes. Eur J Immunol (2000) 30:2540-7. doi:10.1002/1521-4141(200009)30:9<2540::AIDIMMU2540>3.0.CO;2-S
9. Hanahan D, Weinberg RA. Hallmarks of cancer: the next generation. Cell (2011) 144:646-74. doi:10.1016/j.cell.2011.02.013

10. Cobbs CS, Harkins L, Samanta M, Gillespie GY, Bharara S, King PH, et al. Human cytomegalovirus infection and expression in human malignant glioma. Cancer Res (2002) 62:3347-50.

11. Dziurzynski K, Chang SM, Heimberger AB, Kalejta RF, McGregor Dallas SR, Smit M, et al. Consensus on the role of human cytomegalovirus in glioblastoma. Neuro Oncol (2012) 14:246-55. doi:10.1093/neuonc/nor227

12. Söderberg-Nauclér C, Rahbar A, Stragliotto G. Survival in patients with glioblastoma receiving valganciclovir. N Engl J Med (2013) 369:985-6. doi:10.1056/ NEJMc1302145

13. Nair SK, De Leon G, Boczkowski D, Schmittling R, Xie W, Staats J, et al. Recognition and killing of autologous, primary glioblastoma tumor cells by human cytomegalovirus pp65-Specific cytotoxic T cells. Clin Cancer Res (2014) 20:2684-94. doi:10.1158/1078-0432.CCR-13-3268

14. Biggar RJ, Pandey JP, Henle W, Nkrumah FK, Levine PH. Humoral immune response to Epstein-Barr virus antigens and immunoglobulin allotypes in African Burkitt lymphoma patients. Int J Cancer (1984) 33:577-80. doi:10.1002/ ijc. 2910330505

15. Hyöty H, Pandey JP, Lehtinen M, Kulomaa P, Leinikki P. Immunoglobulin allotypes and virus antibodies in Finnish type 1 diabetic patients. Exp Clin Immunogenet (1988) 5:52-9.

16. Pandey JP. Immunoglobulin GM genes and IgG antibodies to cytomegalovirus in patients with systemic sclerosis. Clin Exp Rheumatol (2004) 22:S35-7.

17. Pandey JP, Astemborski J, Thomas DL. Epistatic effects of immunoglobulin GM and KM allotypes on outcome of infection with hepatitis C virus. J Virol (2004) 78:4561-5. doi:10.1128/JVI.78.9.4561-4565.2004

18. Vejbaesya S, Tanwandee T, Pandey JP. Immunoglobulin GM and KM genotypes in hepatitis C virus infection. J Med Virol (2004) 73:384-6. doi:10.1002/ jmv.20102

19. Pandey JP, Montes-Cano MA, Aguilar-Reina J, Gonzalez-Escribano MF. Interactive effects of immunoglobulin gamma and human leucocyte antigen genotypes on clearance and persistence of infection with hepatitis $\mathrm{C}$ virus. Clin Exp Immunol (2007) 150:518-22. doi:10.1111/j.1365-2249.2007.03519.x

20. Pandey JP, Namboodiri AM, Luo Y, Wu Y, Elston RC, Thomas DL, et al. Genetic markers of IgG and the outcome of infection with hepatitis C virus. J Infect Dis (2008) 198:1334-6. doi:10.1086/592282

21. Pandey JP, Luo Y, Elston RC, Wu Y, Philp FH, Astemborski J, et al. Immunoglobulin allotypes influence $\operatorname{IgG}$ antibody responses to hepatitis $\mathrm{C}$ virus envelope proteins E1 and E2. Hum Immunol (2008) 69:158-64. doi:10.1016/j.humimm. 2008.01.019

22. Deepe RN, Kistner-Griffin E, Martin JN, Deeks SG, Pandey JP. Epistatic interactions between $\mathrm{Fc}_{\mathrm{C}}(\mathrm{GM})$ and $\mathrm{Fc} \gamma \mathrm{R}$ genes and the host control of HIV replication. Hum Immunol (2012) 73:263-6. doi:10.1016/j.humimm.2011.12.008

23. Pandey JP, Namboodiri AM, Bu S, Sato A, Dai JY. Immunoglobulin genes and the acquisition of HIV infection in a randomized trial of recombinant adenovirus HIV vaccine. Virology (2013) 441:70-4. doi:10.1016/j.virol.2013.03.007

24. Pandey JP. Genetic and viral etiology of glioblastoma - a unifying hypothesis. Cancer Epidemiol Biomarkers Prev (2011) 20:1061-3. doi:10.1158/1055-9965. EPI-11-0247

25. Pandey JP, Kaur N, Costa S, Amorim J, Nabico R, Linhares P, et al. Immunoglobulin genes implicated in glioma risk. OncoImmunology (2014) 3:e28609. doi:10. 4161/onci.28609

26. Morell A, Scherz R, Käser H, Skvaril F. Evidence for an association between uncommon Gm phenotypes and neuroblastoma. Lancet (1977) 1:23-4.

27. Wolmer-Solberg N, Baryawno N, Rahbar A, Fuchs D, Odeberg J, Taher C, et al. Frequent detection of human cytomegalovirus in neuroblastoma: A novel therapeutic target? Int J Cancer (2013) 133:2351-61. doi:10.1002/ijc.28265

28. Pandey JP, Kistner-Griffin E, Iwasaki M, Bu S, Deepe R, Black L, et al. Genetic markers of immunoglobulin $\mathrm{G}$ and susceptibility to breast cancer. Hum Immunol (2012) 73:1155-8. doi:10.1016/j.humimm.2012.07.340

29. Harkins LE, Matlaf LA, Soroceanu L, Klemm K, Britt WJ, Wang W, et al. Detection of human cytomegalovirus in normal and neoplastic breast epithelium. Herpesviridae (2010) 1:8. doi:10.1186/2042-4280-1-8

30. Taher C, de Boniface J, Mohammad AA, Religa P, Hartman J, Yaiw KC, et al. High prevalence of human cytomegalovirus proteins and nucleic acids in primary breast cancer and metastatic sentinel lymph nodes. PLoS One (2013) 8:e56795. doi:10.1371/journal.pone.0056795 
31. Pandey JP, Kistner-Griffin E, Radwan FF, Kaur N, Namboodiri AM, Black L, et al. Immunoglobulin genes influence the magnitude of humoral immunity to cytomegalovirus glycoprotein B. J Infect Dis (2014). doi:10.1093/infdis/jiu367

32. Pandey JP, Namboodiri AM, Kistner-Griffin E, Iwasaki M, Kasuga Y, Hamada GS, et al. Racially restricted contribution of immunoglobulin $F_{c} \gamma$ and $F c \gamma$ receptor genotypes to humoral immunity to human epidermal growth factor receptor 2 in breast cancer. Clin Exp Immunol (2013) 171:273-7. doi:10.1111/cei.12018

33. Pandey JP, Nietert PJ, Klaamas K, Kurtenkov O. A genetic variant of immunoglobulin $\gamma 2$ is strongly associated with natural immunity to mucin 1 in patients with breast cancer. Cancer Immunol Immunother (2009) 58:2025-9. doi:10.1007/s00262-009-0709-4

34. Torres M, Fernandez-Fuentes N, Fiser A, Casadevall A. The immunoglobulin heavy chain constant region affects kinetic and thermodynamic parameters of antibody variable region interactions with antigen. J Biol Chem (2007) 282:13917-27. doi:10.1074/jbc.M700661200

35. Namboodiri AM, Pandey JP. Differential inhibition of trastuzumab and cetuximab induced cytotoxicity of cancer cells by IgG1 expressing different GM allotypes. Clin Exp Immunol (2011) 166:361-5. doi:10.1111/j.1365-2249.2011. 04477.x

36. Pandey JP, Namboodiri AM. Genetic variants of IgG1 antibodies and Fc $\gamma$ RIIIa receptors influence the magnitude of antibody-dependent cell-mediated cytotoxicity against prostate cancer cells. OncoImmunology (2013) 2:e27317.

37. Pandey JP, Namboodiri AM. Immunoglobulin GM and Fc $\gamma$ RIIIa genotypes influence cytotoxicity of neuroblastoma cells. J Neuroimmunol (2014) 270:95-7. doi:10.1016/j.jneuroim.2014.03.003

38. Cortese M, Calò S, D’Aurizio R, Lilja A, Pacchiani N, Merola M. Recombinant human cytomegalovirus (HCMV) RL13 binds human immunoglobulin G Fc. PLoS One (2012) 7:e50166. doi:10.1371/journal.pone.0050166

39. Moore JH. The ubiquitous nature of epistasis in determining susceptibility to common human diseases. Hum Hered (2003) 56:73-82. doi:10.1159/000073735

40. Phillips PC. Epistasis - the essential role of gene interactions in the structure and evolution of genetic systems. Nat Rev Genet (2008) 9:855-7. doi:10. $1038 / \mathrm{nrg} 2452$
41. Lehner B. Molecular mechanisms of epistasis within and between genes. Trends Genet (2011) 27:323-31. doi:10.1016/j.tig.2011.05.007

42. Jackson SE, Mason GM, Wills MR. Human cytomegalovirus immunity and immune evasion. Virus Res (2011) 157:151-60. doi:10.1016/j.virusres.2010.10. 031

43. Tang J, Shao W, Dorak MT, Li Y, Miike R, Lobashevsky E, et al. Positive and negative associations of human leukocyte antigen variants with the onset and prognosis of adult glioblastoma multiforme. Cancer Epidemiol Biomarkers Prev (2005) 14:2040-4. doi:10.1158/1055-9965.EPI-05-0136

44. Venstrom JM, Zheng J, Noor N, Danis KE, Yeh AW, Cheung IY, et al. KIR and HLA genotypes are associated with disease progression and survival following autologous hematopoietic stem cell transplantation for high-risk neuroblastoma. Clin Cancer Res (2009) 15:7330-4. doi:10.1158/1078-0432.CCR-09-1720

45. Jeong S, Park S, Park BW, Park Y, Kwon OJ, Kim HS. Human leukocyte antigenG (HLA-G) polymorphism and expression in breast cancer patients. PLoS One (2014) 9:e98284. doi:10.1371/journal.pone.0098284

Conflict of Interest Statement: The author declares that the research was conducted in the absence of any commercial or financial relationships that could be construed as a potential conflict of interest.

Received: 23 June 2014; accepted: 16 August 2014; published online: 29 August 2014. Citation: Pandey JP (2014) Immunoglobulin GM genes, cytomegalovirus immunoevasion, and the risk of glioma, neuroblastoma, and breast cancer. Front. Oncol. 4:236. doi: $10.3389 /$ fonc. 2014.00236

This article was submitted to Molecular and Cellular Oncology, a section of the journal Frontiers in Oncology.

Copyright (C) 2014 Pandey. This is an open-access article distributed under the terms of the Creative Commons Attribution License (CC BY). The use, distribution or reproduction in other forums is permitted, provided the original author(s) or licensor are credited and that the original publication in this journal is cited, in accordance with accepted academic practice. No use, distribution or reproduction is permitted which does not comply with these terms. 